\title{
Simulating the fluid forces and fluid-elastic instabilities of a clamped-clamped cylinder in turbulent axial flow
}

\author{
Jeroen De Ridder ${ }^{\mathrm{a}}$, Olivier Doaréc ${ }^{\mathrm{c}}$ Joris Degroote $^{\mathrm{a}}$, Katrien Van Tichelen ${ }^{\mathrm{b}}$, Paul Schuurmans ${ }^{\mathrm{b}}$, Jan Vierendeels ${ }^{\mathrm{a}}$ \\ ${ }^{a}$ Department of Flow, Heat and Combustion Mechanics, Faculty of Engineering and Architecture, Ghent University, Ghent, Belgium \\ ${ }^{b}$ Belgian Nuclear Research Centre, Mol, Belgium \\ ${ }^{c}$ ENSTA - ParisTech, Paris, France
}

\begin{abstract}
In this article, the fluid forces and the dynamics of a flexible clamped-clamped cylinder in turbulent axial flow are computed numerically. In the presented numerical model, there is no need to tune parameters for each specific case or to obtain coefficients from experiments. The results are compared with the dynamics measured in experiments available in literature. The specific case studied here consists of a silicone cylinder mounted in axial water flow. Computationally it is found that the cylinder loses stability first by buckling. The threshold for buckling is in quantitative agreement with experimental results and weakly-nonlinear theory. At higher flow speed a fluttering motion is predicted, in agreement with experimental results. It is also shown that even a small misalignment between the flow and the structure can have a significant impact on the dynamical behavior. To provide insight in the results of these fluid-structure interaction simulations, forces are computed on rigid inclined and curved cylinders, showing the existence of two different flow regimes. Furthermore it is shown that the inlet turbulence state has a non-negligible effect on these forces and thus on the dynamics of the cylinder.
\end{abstract}

Keywords: flow-induced vibrations, fluid-structure interaction, eigenmode, numerical simulation, axial flow

\section{Introduction}

Interest in the simulation and prediction of the dynamics of a flexible cylinder in turbulent axial flow stems from a need to understand the underlying mechanism of flow-induced vibration in a number of engineering applications, such as nuclear reactor cores (Sigrist et al. (2006)) and devices for energy harvesting (Singh et al. (2012)). Research on movement-induced fluid effects on structures dates back to Stokes (1843), who derived an expression for added mass between two concentric cylinders.

The first comprehensive models to predict the dynamics and the onset of instabilities of cylinders in axial flow are the linear models by Païdoussis (1973) and Chen (1987). A review on these models can be found in Païdoussis (2004). The fluid forces in these models are split in a viscous and an inviscid contribution. The inviscid contribution was derived by Lighthill (1960), while the viscous contribution is based on an empirical description (Taylor (1952), Hoerner (1965)). Linear models based on this analysis are still widely used (Sakuma et al. (2008), Rinaldi and Païdoussis (2012))

When applied to the case of a clamped-clamped cylinder, which is also studied in the present work, the linear theories provide a good description of the natural frequency of the cylinder in the stable regime as described by Chen (1987). The damping predicted can be tuned by changing the viscous coefficients in this theory. Regarding instabilities, they provide a good estimation for the onset of divergence. The prediction of fluttering instabilities for a clamped-clamped cylinder is however very dependent on friction coefficients (Païdoussis (1973)).

To improve the accuracy of the linear theory, research on the friction forces perpendicular to the cylinder is still ongoing (Ersdal and Faltinsen (2006); Divaret et al. (2014)). One of the major findings of the latter work is that the so-called independence principle does not hold at low angles of attack. This principle states that the normal force on an inclined cylinder equals the force induced by the cross-flow component of the flow only. However, Divaret et al. (2014) showed that this is only true for relatively high angles of attack $\left(>10^{\circ}\right)$. In this region the lift force 
Table 1: Geometrical parameters of the cylinder (m)

\begin{tabular}{lll}
\hline$D_{o}$ & $D_{i}$ & $L$ \\
\hline 0.0156 & 0.0094 & 0.435 \\
\hline
\end{tabular}

is proportional to the square of the sine of the yaw angle. At low yaw angles, they showed that the lift force varies linearly with the sine of the yaw angle.

In order to examine the post-critical behavior and to verify the validity of linear theory, a weakly nonlinear theory was developed, first for cantilevered cylinders in a three-article publication (Lopes et al. (2002); Païdoussis et al. (2002); Semler et al. (2002)). A similar analysis was later performed for very long cylinders by de Langre et al. (2007). Dynamics for a vanishing stiffness can be found in Triantafyllou (1985). The weakly nonlinear theory (Semler et al. (2002)) was later applied to pinned-pinned and clamped-clamped cylinders (Modarres-Sadeghi et al. (2007)). This theory showed, just like the linear theory, an existence of flutter motion. In contrast to linear theory, it predicts a fluttering motion around a buckled state and not around the neutral axis. To verify the outcome of nonlinear theory, an additional series of experiments were performed by Modarres-Sadeghi et al. (2008). The results of the experiments were in qualitative agreement with the nonlinear theory. However, the threshold at which flutter is predicted disagrees with $40 \%$ and the fluttering motion was also observed around the neutral axis and not around a buckled state.

To improve the accuracy of the predictions in confined annular flow, some computational research was performed as well. Belanger et al. (1994) e.g. developed a model to investigate numerically the dynamics of cylinders in laminar annular flow and Perotin and Granger (1997) developed a linear computational model for a cylinder in turbulent annular flow. An alternative method to these fluid-structure interaction models is to compute the force coefficients required by linear and nonlinear theory from a CFD-calculation (which is e.g. performed by Phan et al. (2013). The drawback is that the force coefficient formulation limits the possible gain of accuracy. This can be remedied by imposing a motion on the structure and computing the modal matrices directly (Facci and Porfiri, 2013). Some contributions, such as damping (Chen, 1987) can however be dependent on frequency of motion, which introduces uncertainty in the results.

In contrast to the previously mentioned theories, De Ridder et al. (2013) developed a methodology, based on coupled computational fluid dynamics (CFD) and structural mechanics computations, to compute modal characteristics of a structure in contact with a (turbulent) flow, without using empirical force coefficients. The method was however only applied for subcritical conditions. The goal of this contribution is to compute the dynamics in a broader range of flow velocities (including the unstable regime) of the clamped-clamped cylinder in turbulent axial flow of Modarres-Sadeghi et al. (2008).

The next section presents a description of the experiment as well as a description of the methods used. To provide insight in the behavior of the viscous forces, simulations on rigid inclined and curved geometries are performed. The results of which are useful for the interpretation of the dynamics predicted by the coupled simulations in the following part. Firstly, the subcritical dynamics are discussed, followed by an analysis of the buckling regime and finally the post-divergence regime is treated.

\section{Description of the experiment and general behavior of a clamped-clamped cylinder in axial flow}

The experimental set-up in Modarres-Sadeghi et al. (2008) consists of a water loop in which a transparent test section with a length of $75 \mathrm{~cm}$ and a diameter of $20 \mathrm{~cm}$ is mounted vertically. It is assumed that a flat velocity profile exists $2.5 \mathrm{~cm}$ away from the test section walls. Information on turbulence levels is however not available. In this test section a (very) flexible, hollow, cylinder is mounted, consisting of silicone rubber. The geometrical parameters, being the outer cylinder diameter $\left(D_{o}\right)$, the inner cylinder diameter $\left(D_{i}\right)$ and the length $(L)$ are provided in Table 1 .

Generally, the system behaves as follows (see Figure 1): at low flow velocities vibrations are small and the cylinder remains straight on average. As the flow velocity increases, vibrations due to turbulence become more important and the amplitude of the vibrations increases. Concurrently the natural frequency decreases (as the destabilizing centrifugal fluid force becomes more important). At a given velocity, the cylinder will buckle in a ground mode shape and show small-amplitude vibrations around this buckled state (due to turbulence). The amplitude of the buckling increases with increasing flow velocity. The onset of buckling is also marked by a vanishing ground mode frequency. 

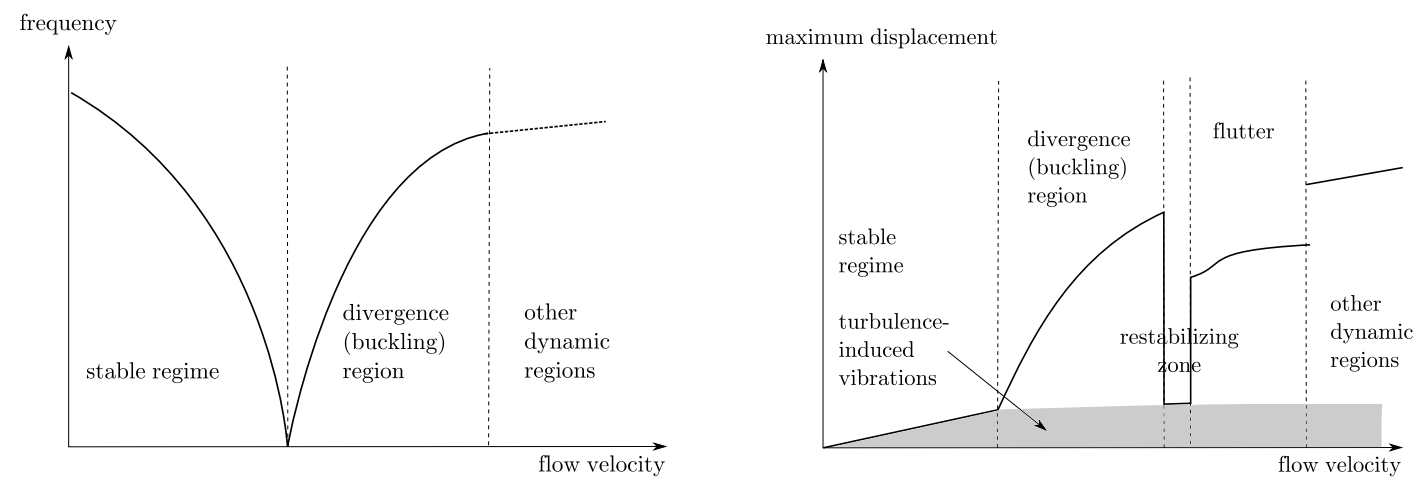

Figure 1: Sketch illustrating the different dynamic zones. The left figure shows the change in eigenfrequency with increasing flow velocity and the right one the maximal amplitude of the cylinder's vibrations as a function of flow velocity.

At even higher flow velocities, the cylinder starts to flutter around its initial straight position. Eventually, there is a small regime of restabilization in between the flutter and divergence (buckling) regime. Note that the maximal displacement in Figure 1 consists of the sum of steady and unsteady contributions.

The onset of divergence can be deduced from the experimental values by using three different methods (ModarresSadeghi et al. (2008)). The first method consists of drawing a parabolic curve through the experimentally measured frequencies. The crossing with the $\mathrm{x}$-axis (zero-frequency) will provide the buckling point. The second method is to look at the measured displacement and draw two straight lines. One in the low velocity range and one in the buckling range. The intercept of the two lines indicates the departure from turbulence-induced vibrations to a buckling instability. The final and most pragmatic approach consists of putting a displacement threshold above which the cylinder is considered unstable. Needless to say, all three methods suffer from inaccuracies, yet they all provide useful information.

\section{Methodology}

In this paper, the modal characteristics of a cylinder in the unstable region are determined with the methodology published earlier in De Ridder et al. (2013). Briefly summarized, this methodology consists of four steps:

1. Compute in-vacuum eigenmodes

2. Deform solid and fluid domain with scaled eigenmode

3. Compute the free vibration decay $(w(z, t))$

4. Fit a modal expression to the computed decay $\left(w(z, t) \approx \sum a_{i}(z) e^{-c_{i} t} \sin \left(\omega_{i} t+\phi_{i}(z)\right)\right)$

Initially, the in-vacuum eigenmodes of the structure are calculated. These modes are used to deform the solid and the fluid domain. The deformed solid and fluid domain serve as initial conditions for a coupled computational fluid dynamics (CFD) - computational solid mechanics (CSM) computation using a partitioned but fully coupled approach (Degroote et al., 2009), in which the free vibration decay is thus computed. The final step is the fitting of a modal expression to the computed decay. Note that the initially imposed in-vacuum mode of the structure alone does not necessarily agree with the modes of the fluid and structure together.

In the CFD-part, the Reynolds-averaged Navier-Stokes equations are solved, where the turbulence is modeled by the k- $\omega$ SST model of Menter (1994). As turbulent eddies are not explicitly resolved, it is impossible to predict the turbulence-induced vibrations part in Figure 1 with the present computations. As inlet conditions, a very low inlet turbulence level is applied, so that turbulence in the flow is generated by the developing boundary layer on the cylinder. The influence of inlet turbulence intensity is explicitly checked in sections 4.1 and 7.2. The equations are all of second-order accuracy, both in time and in space. The CSM-computations are performed with quadratic solid elements and the backward Euler time integrator. The CFD-code used throughout this work is Fluent 14.5, Ansys Inc., the CSM-code Abaqus 6.12, Simulia and the coupling code is an in-house code, employing the IQN-ILS algorithm, as described in Degroote et al. (2009). 

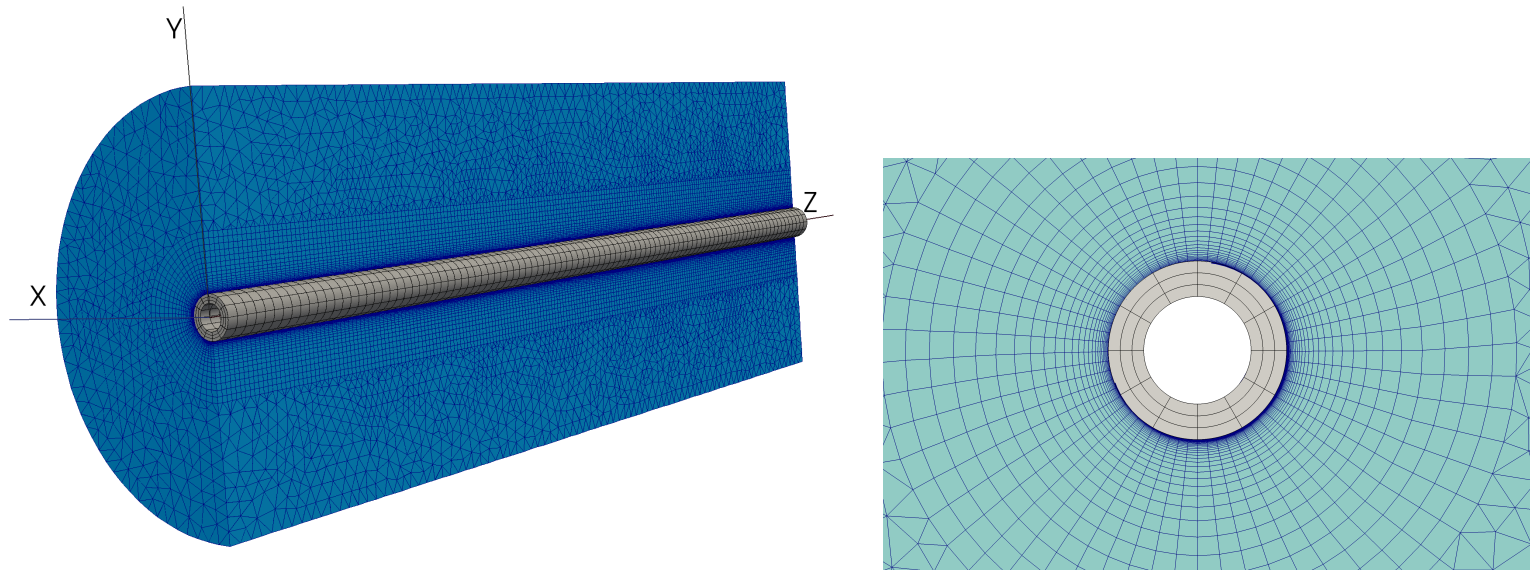

Figure 2: Cross-sectional view of the meshes, with the solid mesh in grey and the fluid mesh in blue.

The grids used for the simulations are plotted in Figure 2. The construction of the meshes is based on a previous convergence study in De Ridder et al. (2013). Furthermore, computing lift forces on a rigid but tilted geometry with the standard mesh and a mesh twice finer in all directions, so with eight times more degrees of freedom, yielded forces with only two percent difference. Coupled simulations with the standard and a coarser mesh provided similar results, except for the fluttering range, which is sensitive to even small changes in lift force.

The parameters which are used throughout the simulations are listed in Table 2. The non-dimensionalization used is the same one as in Modarres-Sadeghi et al. (2008):

$$
\beta=\frac{\rho A_{f}}{\rho A_{f}+m}, \Gamma=\frac{T L^{2}}{E I}, \epsilon=\frac{L}{D_{o}}, u=\left(\frac{\rho A_{f}}{E I}\right)^{1 / 2} \mathrm{v} L, h=\frac{D_{o}}{D_{h}}, \Pi_{0}=\frac{E A_{s} L^{2}}{E I},
$$

in which $\rho$ is the fluid density, $\rho A_{f}$ the added mass of the fluid per unit length, with $A_{f}$ the cross-section of the cylinder in the fluid $\left(=\pi D_{o}^{2} / 4\right), m$ the cylinder's mass per unit length, $T$ the external tension applied on the cylinder, $E$ the Young's modulus, $I$ the area moment of inertia, v the mean axial flow speed, $L$ the cylinder's length, $D_{o}$ the outer diameter of the cylinder, $D_{h}$ the hydraulic channel diameter and $A_{s}$ the cross-sectional area of the cylinder $\left(=\pi\left(D_{o}^{2}-D_{i}^{2}\right) / 4\right)$. The reader will notice that gravity and pressurization are left out as they are both taken zero. It was verified by linear theory that these parameters had, for the range of conditions studied in this contribution, limited effect.

Three additional parameters are however included: the inlet turbulence intensity $(T I)$, a non-dimensional characteristic inlet turbulence length scale $(N T L S)$ and the Reynolds number of the fluid $\left(\operatorname{Re}_{D}\right)$ :

$$
N T L S=\frac{T L S}{L}, T I, R e_{D}=\frac{\rho v D_{o}}{\mu},
$$

with $T L S$ the turbulent length scale and $\mu$ the fluid's viscosity. Those parameters were not specified in ModarresSadeghi et al. (2008), but they are necessary to characterize the simulations.

In the following graphs, changes of fluid velocity will be studied, which are reported as changes in non-dimensional flow velocity $(u)$. It should however be taken into account that the Reynolds number is changing concurrently, except for the computations on a rigid geometry. The influence of the Reynolds number is studied separately on a rigid geometry (which has an infinite stiffness and thus $\mathrm{u}=0$, while Re changes). The Poisson ratio ( $v$ ) is chosen to be 0.4 , which is slightly lower than characteristic values for silicone rubber. However, this value becomes only important for high shearing rates or when significant radial loading is placed on the rubber. 


\section{Fluid forces on rigid geometry}

Before the results of a deforming cylinder coupled with the surrounding flow are presented, the fluid forces are computed on rigid but deformed structures. Two types of deformation are considered: a tilted geometry and a curved one. The computations on a tilted geometry are similar to the computations and experiments done by Divaret et al. (2014). As a steady inviscid simulation gives zero forces for an inclined cylinder, the lift forces in the viscous simulations are named viscous forces in the remainder of the text although they are mainly composed of pressure contributions.

\subsection{Yawed cylinder}

The interest of performing simulations on tilted or yawed geometries is that the resulting forces and force coefficients are similar to the forces which generate damping on a clamped-clamped cylinder in axial flow. In the experiments of Ersdal and Faltinsen (2006) and Divaret et al. (2014) it was found that at low angles of attack the lift force varies linearly with angle of attack, while at higher angles of attack the cross-flow principle holds, which predicts a quadratic angle dependency.

The main contribution towards the lift force stems from the pressure in the fluid. Its distribution approximately halfway along the cylinder is plotted in Figure 3 for different angles of attack. At the lowest angle, the pressure profile is very close to a pressure profile governed by inviscid forces. There is a partial stagnation point at the down side of the cylinder. Left and right from this point, the flow accelerates to flow over the cylinder, with the related pressure decrease. After the maximum deflection, the flow decelerates, and the pressure rises again. The resulting pressure profile is however modified by the presence of the boundary layers, which induce a pressure difference between top and bottom of the cylinder.

At a higher angle, $5^{\circ}$ in Figure 3, two areas of low pressure on top of the circle appear. These low-pressure areas are linked to the appearance of two stable counter-rotating vortices. As the angle increases further these vortices become more pronounced and the separation, which causes the vortices, dominates the pressure profile at the back of the cylinder. Consistently with the experimental results of Divaret et al. (2014), the pressure on the front side of the cylinder is highest, decreasing rapidly as the flow accelerates over the circle.

The standard force coefficients are defined as follows:

$$
C_{i}=\frac{\int_{z_{0}}^{z_{1}} F_{i} \mathrm{~d} z}{1 / 2 \rho \mathrm{v}^{2} D_{o}\left(z_{1}-z_{0}\right)},
$$

with $F_{i}$ the force per meter cylinder length in direction $i, z$ the axial coordinate and $z_{0}, z_{1}$ the end points of integration. To avoid effects of boundary conditions, the force is computed on a section of $0.2 \mathrm{~m}$ (about half of the cylinder's length), which has the midpoint of the cylinder as center. If the integration interval is split in two, the lift force on the upstream half is $10 \%$ lower than on the downstream half on the cylinder inclined at $1^{\circ}$ and with a $1 \mathrm{~m} / \mathrm{s}$ flow speed. This indicates that there is little axial variation in the force distribution.

As the results in Divaret et al. (2014) show that the lift coefficient varies linearly with the angle of attack $\left(C_{L}=\right.$ $\left.C_{l, \alpha} \alpha\right)$ at low angles $\left(<5^{\circ}\right)$, the ratio of the computed lift coefficient over the angle in radians is plotted for different angles of attack and different Reynolds numbers (changing flow velocities, constant material properties) in Figure 4. The left figure shows that this ratio is indeed nearly constant up to 1 degree. At higher angles of attack it follows a different trend. This behavior of force coefficient is in line with the flow profiles, which showed that at higher angles, a significant double vortex develops. At lower angles of attack, the double vortex is not present, as shown on Figure 3 , resulting in a different lift force.

As the turbulent flow in these simulations is not fully developed it is likely that the inlet flow conditions will affect the friction considerably. To quantify this influence, a variation of inlet turbulence intensity from $0.1 \%$ to 10

Table 2: Non-dimensional parameters of the simulations.

\begin{tabular}{lllllllllll}
\hline & $\beta$ & $\Gamma$ & $\epsilon$ & $u$ & $v$ & $T I(\%)$ & $N T L S$ & $h$ & $\operatorname{Re}_{D}$ & $\Pi_{0}$ \\
\hline rigid & 0 & 0 & 27.88 & 0 & 0.4 & $0.1-10$ & $0.0023-0.023$ & 0.0768 & $15600-156000$ & 9124.2 \\
flexible & 0.57 & 1.21 & 27.88 & $3.0-11.25$ & 0.4 & 0.05 & 0.0023 & 0.0768 & $19800-74400$ & 9124.2 \\
\hline
\end{tabular}


$1^{\circ}$
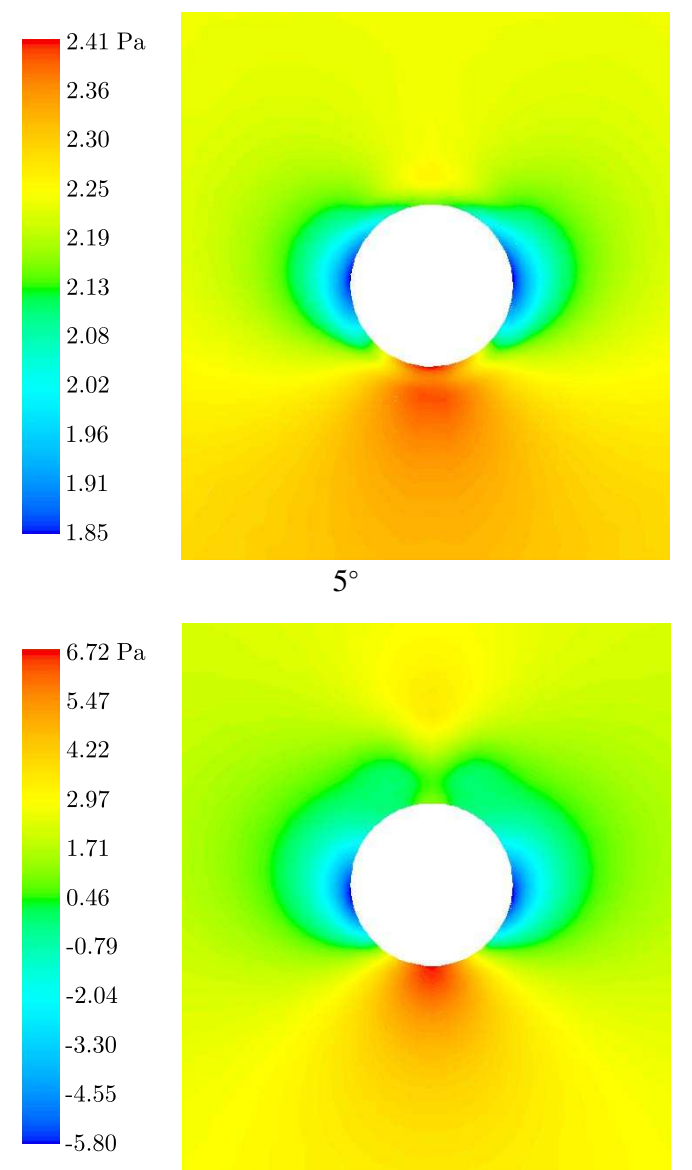
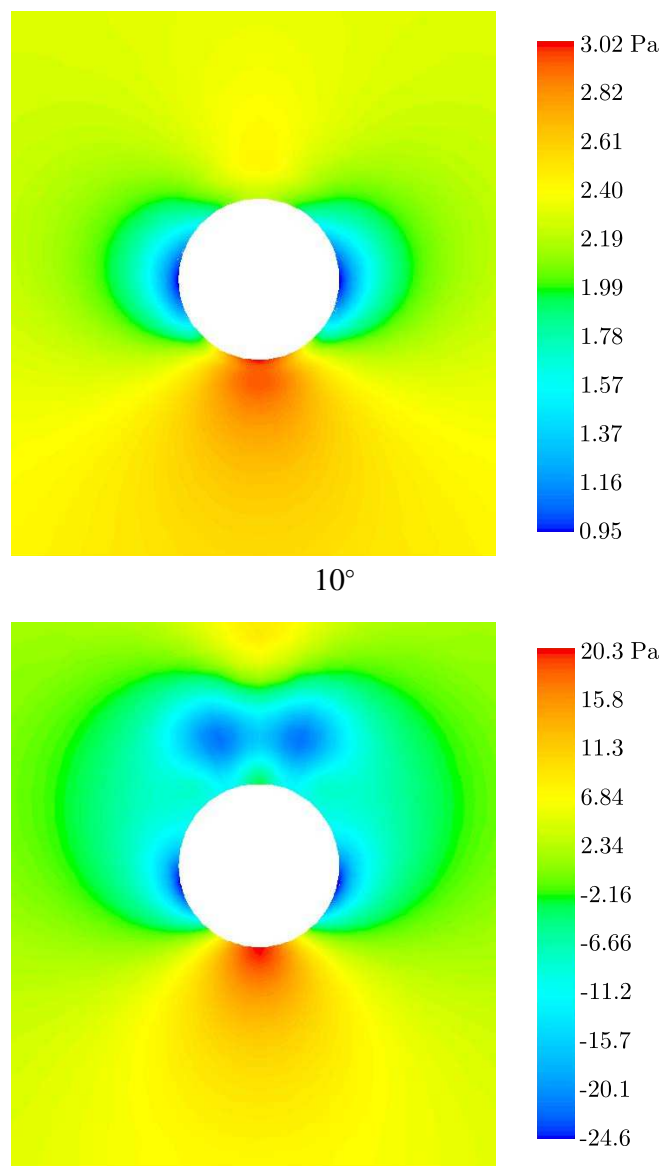

Figure 3: Pressure distribution $(\mathrm{Pa})$ on a plane at $\mathrm{z}=0.2 \mathrm{~m}$ for a cylinder tilted in such a way that a negative slope is obtained. The cylinder is rotated by $1^{\circ}, 2^{\circ}, 5^{\circ}$ and $10^{\circ}$.

$\%$ and inlet turbulence length scale from 0.001 to $0.01 \mathrm{~m}$ is performed, corresponding to a change from low to highly turbulent inlet flow. The lift coefficient as a function of turbulence parameters and angle of attack is shown in Figure 4a. At a low angle of attack, the lift coefficient is seen to vary considerably with the inlet turbulence intensity. At high angles of attack however, the inlet turbulence has less effect. Again, the different behavior can be explained by the flow mechanism. Where the lift coefficient at low angles of attack depends on the pressure loss in the flow, it depends for higher angles mainly on the separation characteristics, which are less dependent on the main flow turbulence intensity.

As the lift coefficient depends on the inlet turbulence, it is quite difficult to make a comparison of the current lift coefficient with coefficients found in literature. Comparing the constant factors $\left(C_{L} \alpha^{-1}\right)$ found in this work (0.02-0.2) to the one in Divaret et al. (2014) (0.11) or the one of Ersdal and Faltinsen (2006) (0.068), it is seen that the literature values fall within the range of predicted values.

A final remark on the force coefficients is that they are not completely independent from Reynolds number as shown on Figure 4b. From a least-squares curve fit, the influence of the Reynolds number is approximately

$$
C_{L} \propto R e_{D_{o} / \alpha}^{-0.15}
$$

The Reynolds number in this equation is a modified Reynolds number. The length unit used is proportional to the path length of the fluid across the cylinder, which, for low angles of attack, is proportional to $D_{o} / \alpha$ :

$$
R e_{D_{o} / \alpha}=\frac{\rho v D_{o}}{\mu \alpha}
$$


(a)

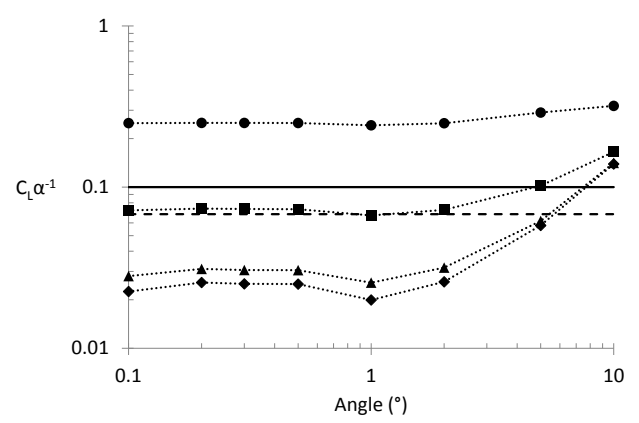

(b)

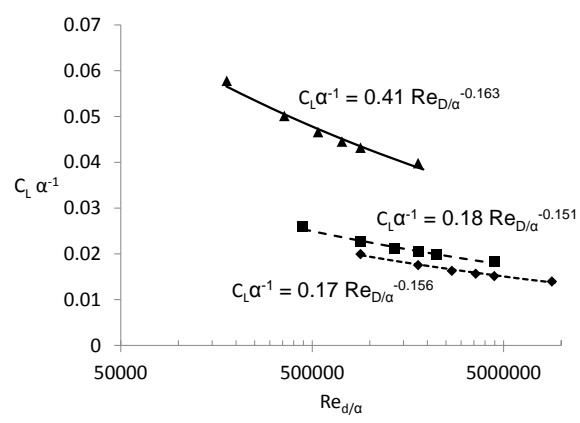

Figure 4: Lift coefficient divided by angle of attack as a function of angle (a) and Reynolds number (b). The Reynolds number $\left(R e_{D}\right)$ in (a) is 15600

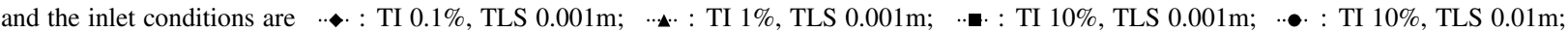
— : value Divaret et al. ; _ - : value Ersdal et al. The turbulence inlet condition in (b) are TI $0.1 \%$ and TLS $0.001 \mathrm{~m}$. The cylinder is rotated at - : $1^{\circ} ; \mathbf{\square}: 2^{\circ}$ and $\boldsymbol{\Delta}: 5^{\circ}$.

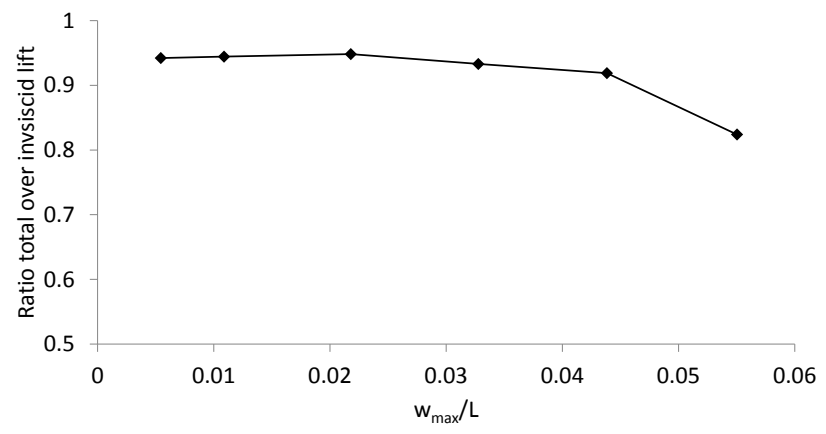

Figure 5: Total lift divided by the inviscid contribution as a function of curvature. This shows that the viscous contribution is counteracting the inviscid force.

\subsection{Curved cylinder}

If the cylinder is clamped at both ends, its motion will be composed of bending movements. The linear inviscid flow reaction exerted locally on a statically curved cylinder reads (Lighthill, 1960):

$$
F_{y, \text { curv }}=A_{f} \rho U^{2} \frac{\partial^{2} w}{\partial z^{2}}
$$

with $w$ the deflection of the cylinder, $z$ the axial coordinate and $A_{f}$ the cross-sectional area immersed in the fluid.

In this section, the cylinder is deformed by circular arcs of different radii. The applied curvature will be reported as the ratio of the maximal deflection over the length of the cylinder $\left(w_{\max } / L\right)$. It is assessed whether a viscous contribution to curvature forces exists. If the viscous forces depend only on local inclination, the forces on the upstream half of the cylinder will cancel out the forces on the downstream part. As a negative curvature is applied, the viscous forces on the upstream half of the cylinder are pointing downwards while the ones downstream are pointing upwards.

Figure 5 shows that low curvatures indeed give rise to a force which is close to the one predicted by inviscid theory. It is however smaller than its inviscid counterpart, meaning that in the viscous simulation there is a force counteracting the upward pointing inviscid force. This means that the viscous forces are stronger upstream, which are pointing downwards, than downstream. At higher curvatures, the total lift force becomes progressively smaller than the inviscid part.

In Figure 6 pathlines of wall shear stress are shown for cylinders deformed with a low and high curvature arc. In this figure, the flow is coming from the left and going to the right. If the flow remains attached, the pathlines on 

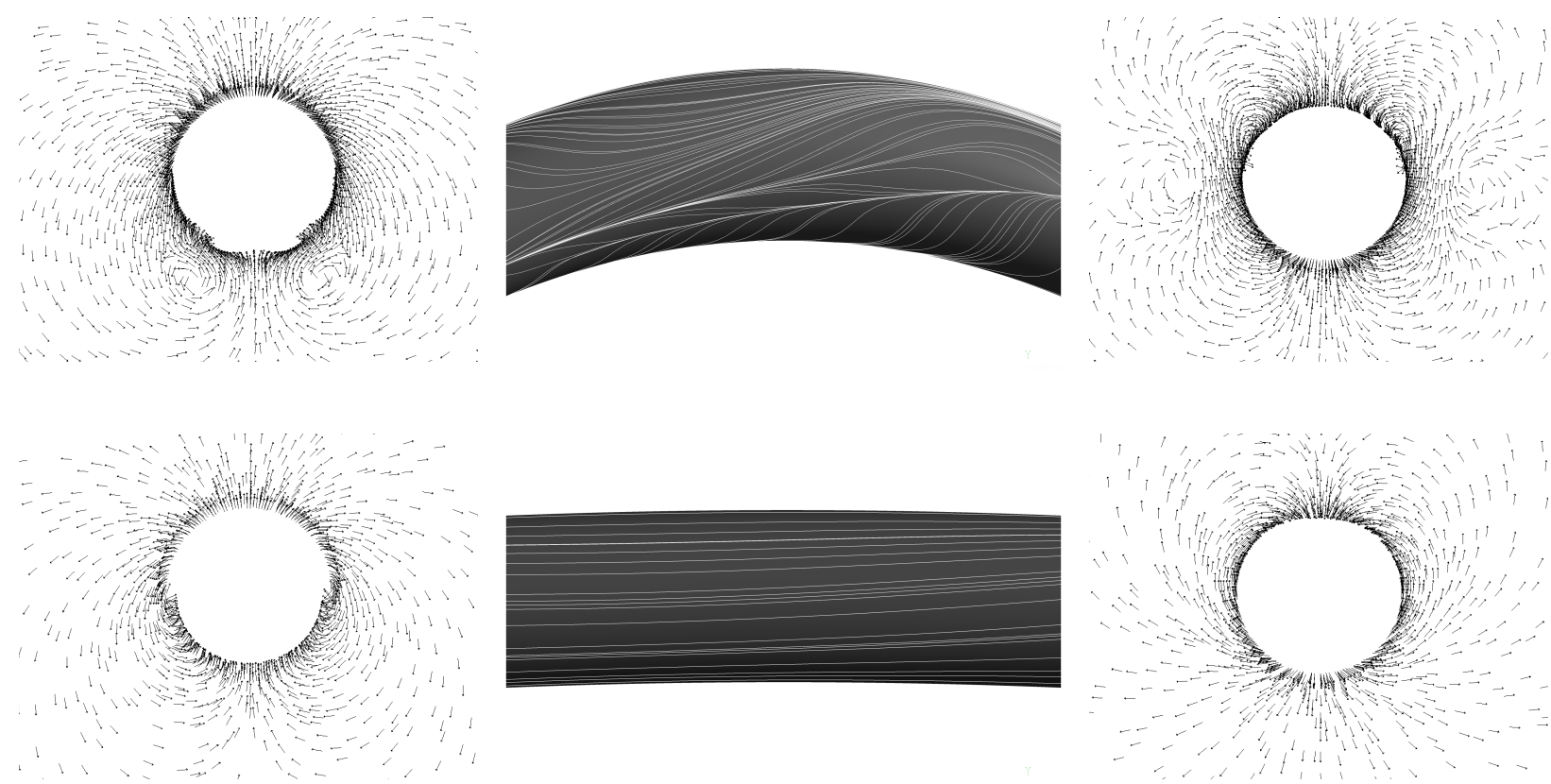

Figure 6: The middle figures display pathlines of wall shear stress on a highly curved cylinder (top figure, $w_{\max / L}=0.055$ ) and moderately curved cylinder (bottom figure, $w_{\max / L}=0.00544$ ). The cylinder displayed is clipped to the part on which the forces are computed. It has been scaled such that the pathlines are visible; in reality the ratio of the cylinder's length over diameter is 27.9 . The left and right figures show the projected velocity vectors, taken at the upstream cross-section (left) and downstream section (right). These figures show the (transported) vortices at high curvature while the flow at low curvature remains attached.

the downside will converge to the bottom of this view. If a double vortex would occur, the pathlines would diverge from the downside of the cylinder. Furthermore, an apparent crossing of pathlines will appear, which is the region of detachment (Lighthill, 1963).

At low curvature $\left(w_{\max / L}=0.00544\right)$, the pathlines show an attached flow, while at high curvature $\left(w_{\max / L}=0.055\right)$ a vortex is present at the left down side of the cylinder in Figure 6. At high curvature, the maximal local angle of attack is $12.5^{\circ}$. If it is high enough, the flow detaches and a vortex develops (see previous section). This separation point and the vortex are then transported downstream even if the downstream angle of attack is not high enough to initiate vortex formation. On the right upper part, this flow does not yet separate. The viscous forces thus affect the curvature-related force, but it is not directly linked to curvature. It rather depends on the combination of local angle and the flow detachment and re-attachment characteristics. The force arising from viscous effects can thus generally not be described by local angle-dependent lift forces only.

\section{Dynamics in stable regime}

In this and the following sections, the results of the coupled simulations are presented. The analysis of dynamics in the stable regime is performed in the same way as in De Ridder et al. (2013). The geometry is deformed with an in-vacuum eigenmode, after which the free vibration of the structure in the flow is computed by coupled simulations. The resulting vibration is then analyzed by fitting modal expressions to it.

The frequency $(f)$, damping $(c)$ and deflection $(w)$ are reported non-dimensionally $\left(_{N D}\right)$ as a function of nondimensional flow velocity. Following Modarres-Sadeghi et al. (2008), they are given by the following formulas:

$$
f_{N D}=L^{2}\left(\frac{m+\rho A_{f}}{E I}\right)^{1 / 2} f, c_{N D}=L^{2}\left(\frac{m+\rho A_{f}}{E I}\right)^{1 / 2} c, w_{N D}=\frac{w}{L} .
$$



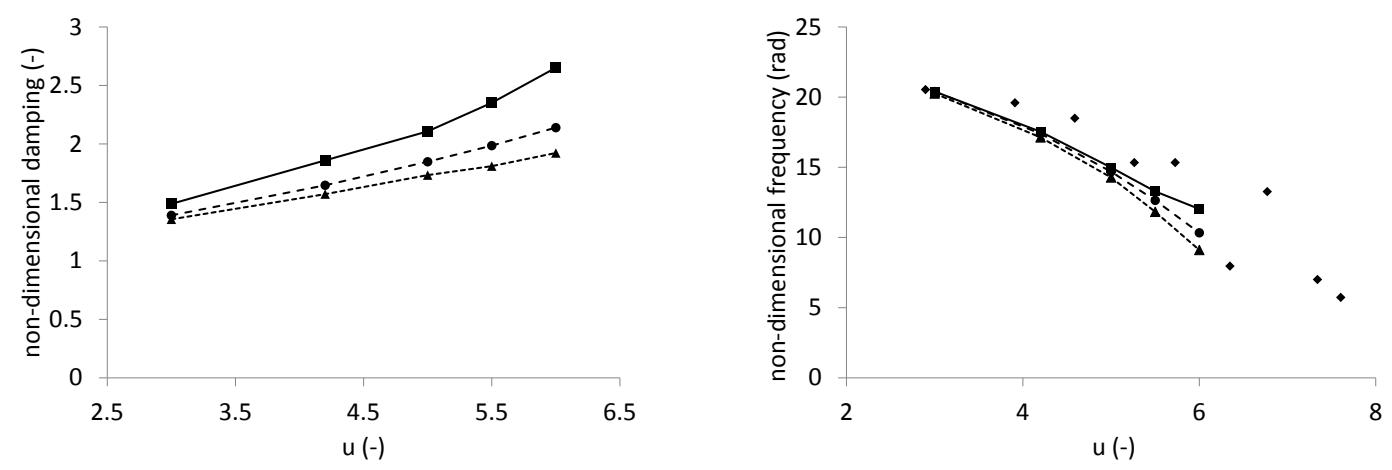

Figure 7: Modal characteristics (damping on the left graph and frequency on the right one) in the stable regime as function of flow velocity. Legend: - : experimental;

The variation of non-dimensional flow velocity was accomplished by changing the mean flow velocity, which also changes the Reynolds number. As shown in Section 4.1, the changing Reynolds number will have a small influence on the lift coefficient.

The computed modal characteristics are reported in Figure 7. Consistent with the linear angle dependency of viscous forces, demonstrated earlier, the damping increases linearly with flow velocity:

$$
F_{y}=C_{y \alpha} \alpha 1 / 2 \rho \mathrm{v}^{2} D_{o}, \alpha \approx \frac{1}{\mathrm{v}} \frac{\partial w}{\partial t} \Rightarrow F_{y}=C_{N \alpha} 1 / 2 \rho \mathrm{v} D_{o} \frac{\partial w}{\partial t} .
$$

The value of the damping presented here is likely to be too low as it depends on the inlet turbulence conditions, which are set to nearly laminar conditions in the present simulations.

The frequency, as expected, decreases with increasing flow velocity. There is reasonable agreement between computed and measured values of Modarres-Sadeghi et al. (2008). The numerical simulations have however tendency to predict slightly lower frequencies. From this graph, the onset of divergence can be determined as the crossing of the curve with the horizontal axis. The resulting non-dimensional buckling velocity equals 6.4.

A comparison of natural frequency of the cylinder at different inclination angles and flow speeds in Figure 7 shows that the frequency of more inclined cylinders decreases a bit slower. Concurrently, the damping increases more for cylinders at larger inclination angles, which can be explained by the small change in friction coefficients, examined in the previous section.

\section{The prediction of divergence}

The analysis of the divergence region is split into three parts. Firstly, the onset of instability is determined, after which the buckled state is examined and finally the transients towards the buckled state are discussed.

\subsection{The onset of divergence}

Three methods for the detection of the onset of divergence were used in Modarres-Sadeghi et al. (2008) to analyze the experimental results: the frequency fitting method (method 1), the displacement fitting method $(\operatorname{method} 2)$ and the displacement threshold method (method 3). Numerically, at least for an initial fully straight geometry, the steady-state deformation in the pre-divergence region will be zero. Provided that the chosen threshold is small enough, this implies that the second and third method will give the same result when applied to the numerical results.

In Figure 8, the maximal displacement of the cylinder at half height is shown for different flow velocities. In the rightmost part of the graph (denoted by gray symbols) the cylinder is fluttering. The figure shows the displacement of the straight cylinder as well as the displacements for the slightly inclined cases. The experimental values are taken directly from Modarres-Sadeghi et al. (2008). They represent the maximal amplitude measured halfway the cylinder. 


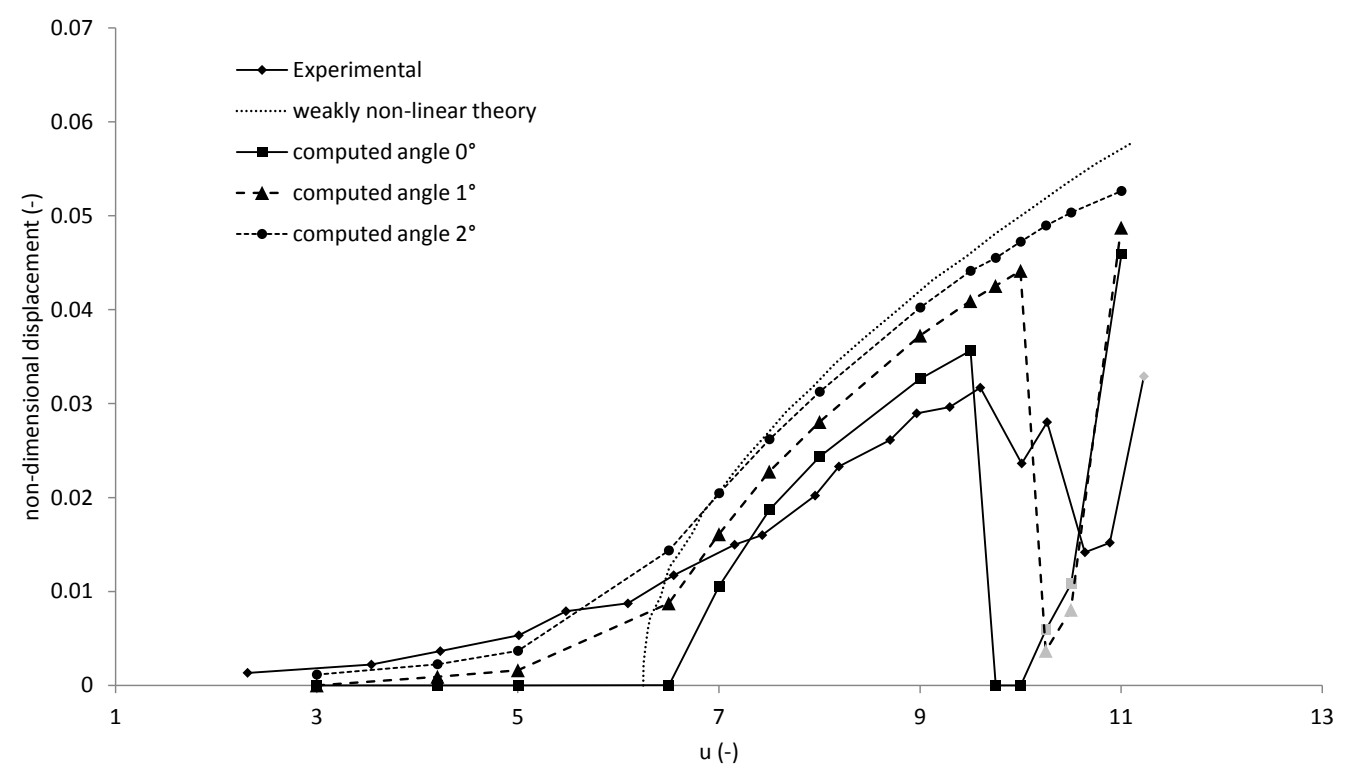

Figure 8: Comparison of the displacement of the cylinder at half height at different flow velocities between simulations, experiments and weakly non-linear theory (Modarres-Sadeghi et al. (2007)). The black symbols denote steady deformation while the gray symbols represent the maximal amplitude of a fluttering motion around the neutral line.

Based on the simulated displacement, the buckling will start at a velocity between $u=6.5$ and 7 . At increasing flow velocity, the deflection will grow. The growth rate seems however to be the largest in the initial region. The maximum deflection was in the same order of magnitude as the cylinder's outer diameter. At a non-dimensional flow velocity of approximately 10 , the cylinder regains stability. The dynamics in that region will be discussed later.

Regarding the onset of buckling, the different measurements are gathered in Table 3. The numerical methods all give approximately the same result, which is that divergence is appearing at a flow velocity around $6-7$.

There is agreement between the experimental results and the numerical ones, although it is difficult to judge due to the scatter on the different experimental values. The agreement between the non-linear theory and the computational results is however very good. This can be understood as the occurrence of divergence is triggered by non-viscous (centrifugal) fluid forces, which are well incorporated in the non-linear theory.

Comparing computational or theoretical results to experimental values is often difficult as uncertainties on both sides exist. One of the uncertainties in this case is whether the cylinder is $100 \%$ aligned with the axial flow. The influence of alignment is therefore studied by putting the cylinder at an angle of attack of one and two degrees. Figure 8 shows that small inclinations might, next to turbulence-induced vibration, explain the experimental low-velocity displacement. Note that the resulting displacement colored in black in Figure 8 is the steady state displacement.

Table 3: Comparison of the onset of divergence, predicted by experiments, weakly non-linear theory and the present simulations by using different methods

\begin{tabular}{lll}
\hline Measurement type & method & critical velocity (-) \\
\hline Experimental & method 1, elliptic fit & 7.7 \\
Experimental & method 1, parabolic fit & 7.5 \\
Experimental & method 2 & 5.4 \\
Experimental & method 3 & 6.2 \\
Non-linear theory & method 1 & 6.25 \\
Computational & method 1 & 6.4 \\
Computational & method 2 & $6.5-7$ \\
\hline
\end{tabular}




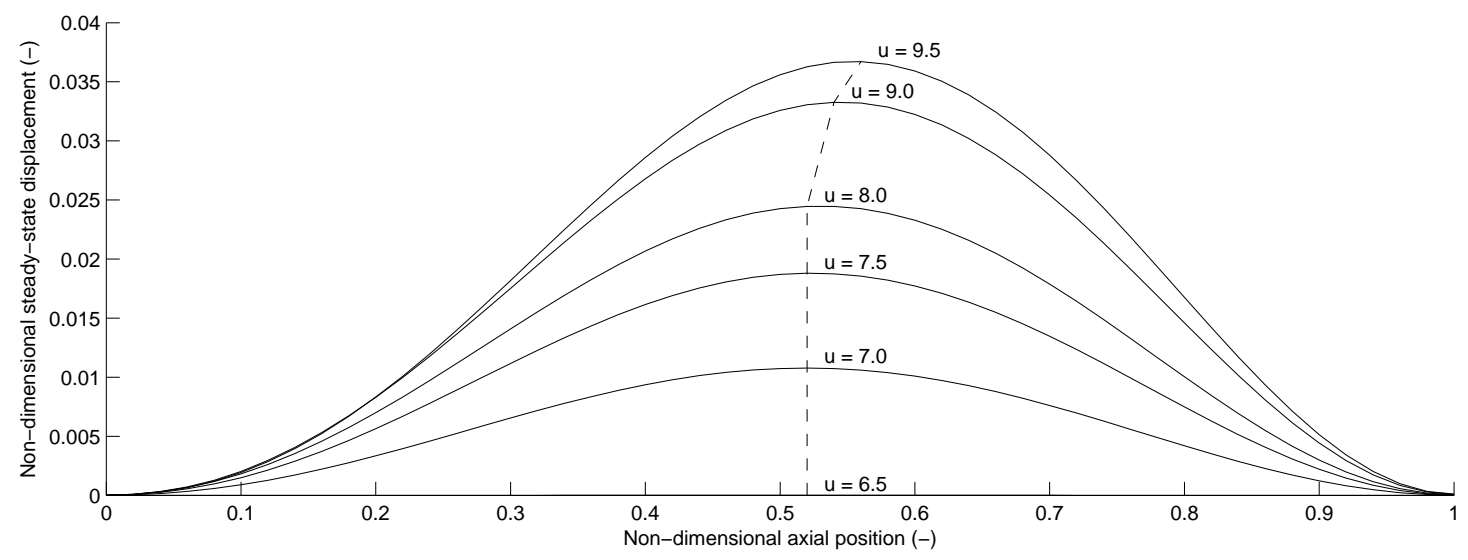

Figure 9: Simulated steady-state displacement of the buckled cylinder at different flow velocities with indication of the downstream shift of the maximal displacement.

Characterizing the onset of divergence from the displacement graph, Figure 8, is now slightly more difficult. It is however seen that the tilting does not have a significant effect on the onset of divergence, although the cylinder is already deformed at lower velocities.

\subsection{The buckled state}

Once the cylinder is buckled, the predicted displacement in Figure 8 corresponds reasonably well with the measured one. At low velocities however, a totally aligned geometry does not show an average displacement. The experimental displacement can be caused by turbulence-induced vibrations or misalignment. The latter influence is tested and it is seen to indeed give rise to similar deformations. At higher flow velocities, a small overshoot of the predictions may be noticed. It may be observed that, even though it is not perfect, the numerical simulations provide more accurate information than the weakly non-linear theory. The improved agreement with experimental values can be explained by the improved viscous force prediction, which was shown to counteract the inviscid destabilizing force.

The buckled states are displayed in Figure 9. As already explained previously, the amplitude of these states increases with increasing flow velocity. Furthermore, in agreement with non-linear theory and experimental observations, the cylinder is buckled in a mode shape, which resembles a first eigenmode shape of a solitary clamped-clamped cylinder. It however differs from the in-vacuum eigenmode shape as the location of the maximum is shifted downstream. This could be attributed to the drag forces acting on the cylinder. This downstream shift is something which is also observed experimentally (Païdoussis, 2004).

The first and predictable effect of putting cylinders at an angle is that the cylinder will deform due to the statically imposed load, even at low flow velocities. A misalignment may thus be one of the contributing factors to the deformation amplitude measured experimentally. Inside the divergence region, the displacements are always bigger for inclined cylinders. This is to be expected as on top of the destabilizing inviscid centrifugal force, an additional viscous contribution is present.

A remarkable change in dynamics is that the onset of restabilization is postponed or even skipped altogether. The first dynamic regime of the cylinder at $1^{\circ}$ is a flutter regime, as can be seen in Figure 8 . At a higher flow velocity, the flutter regime goes back to the divergence regime. If the cylinder is put at an even bigger angle of $2^{\circ}$, the restabilizing and flutter regime are skipped entirely. The resulting dynamics are a further growth of divergence. This might be explained by the following reasoning: Consider the cylinder in its initial straight but inclined position. Even in the absence of fluid-elastic effects, it experiences a fluid force (the lift force in Section 4), in contrast to a non-inclined cylinder. This destabilizing force might be capable of overcoming the fluid-elastic forces that would otherwise cause flutter motions.

The former observations can actually play an important role in the prediction of flow-induced vibration. In this section the influence of a mean inclination on the dynamics is shown. In reality however, the flow is turbulent and the 


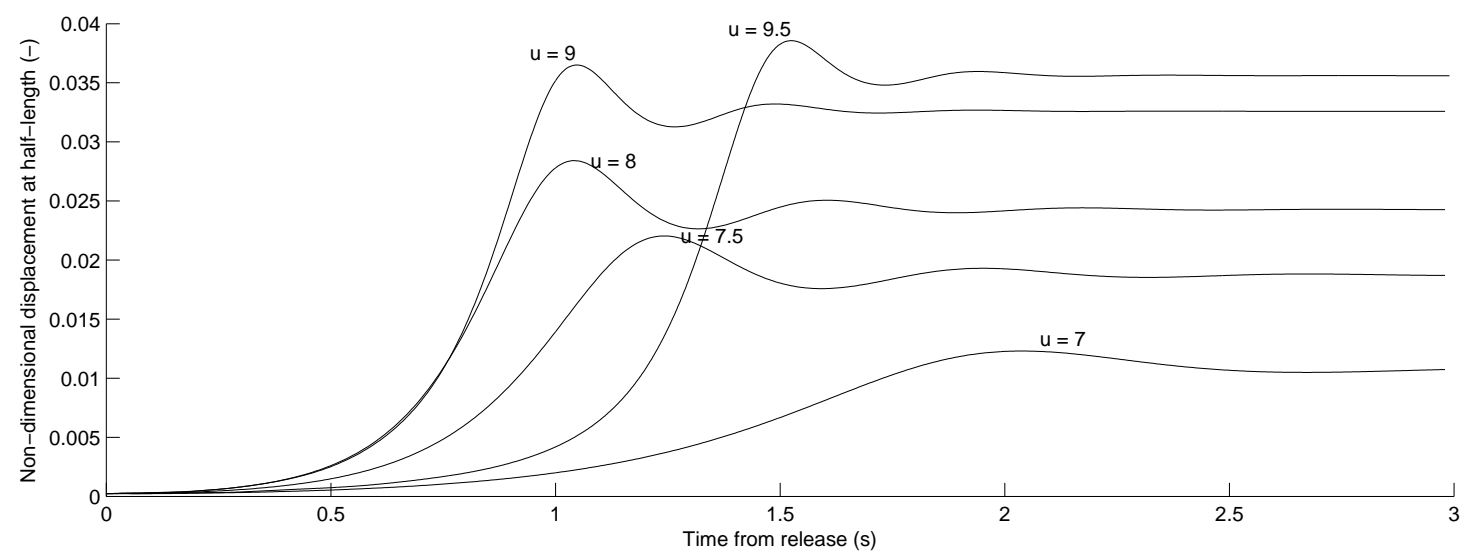

Figure 10: Time history of the computed displacement halfway along the cylinder at different flow velocities.

Table 4: Growth rate in the repelling transient according to the linear theory of Païdoussis (1973) and the presented simulations.

\begin{tabular}{llllll}
\hline $\mathrm{u}(-)$ & 7.0 & 7.5 & 8.0 & 9.0 & 9.5 \\
\hline$c_{N D}(-)$ linear theory & 5.30 & 8.29 & 9.79 & 7.34 & flutter \\
$c_{N D}(-)$ simulations & 3.26 & 6.25 & 7.48 & 6.93 & 4.49 \\
\hline
\end{tabular}

inclination angle of the cylinder with respect to the flow may be changing all the time. If the flow is coherent enough, this means that the response will be a combination of the responses presented in this paragraph.

\subsection{The transient towards the buckled state}

Figure 10 displays the evolution of the center line displacement halfway along the cylinder. In contrast to transients in a stable regime, they are more difficult to interpret. The easiest part is definitely the steady state configuration, which was discussed previously. A tentative analysis splits the transient in two regions: the region where the cylinder moves away from the zero-axis and the settling region around the buckled state.

\subsubsection{Repelling dynamics}

The former region (the repulsive one) is seen to have an initially steeper slope as the flow velocity increases (up to $\mathrm{u}=8$ ), followed by a flattening of the initial slope (from $\mathrm{u}=8$ ). A way of characterizing the repulsive region is to fit an exponential function to all points. This fitting is performed for 20 points distributed uniformly along the cylinder's center line. The time signal is truncated to where it reaches half of its steady-state displacement.

The resulting exponential growth rates are listed in Table 4. In the same table, the predictions according to linear theory of Païdoussis (1973) are also listed. Interestingly, despite the rather basic modeling of viscous forces in the linear theory, the exponential factors are found to be relatively similar. Both the coupled simulations and the linear theory predict an exponentially growing deformation which initially gets faster with increasing flow velocity. At higher flow velocity the exponential growth gets slower, until it will (in this case) restabilize.

To investigate the initial repelling region further, the center line displacements are plotted for $0.5 \mathrm{~s}$ of motion on Figure 11. At the lowest flow velocity at which divergence occurs (Figure 11a), all points are seen to move in phase towards the buckled state. At the highest flow velocity at which divergence is maintained (Figure 11d), the initial deformation is seen to develop in an S-shaped form. This means that the dominant repelling mode which at low velocity resembles a first structural mode shape changed to an S-shaped mode.

The mode shapes of the unstable mode are plotted in Figure 12. There it is seen that both linear theory and the current computations predict the change in mode shape from a nearly first structural mode shape towards a combination of a first and second structural mode. 
(a) $\mathrm{u}=7$

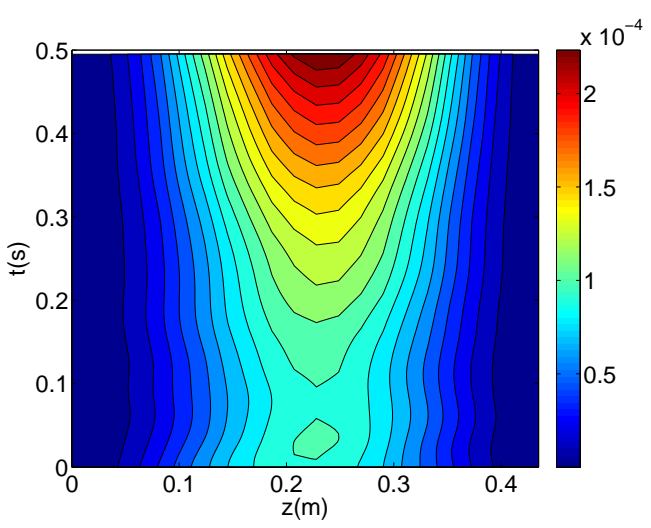

(c) $\mathrm{u}=8$

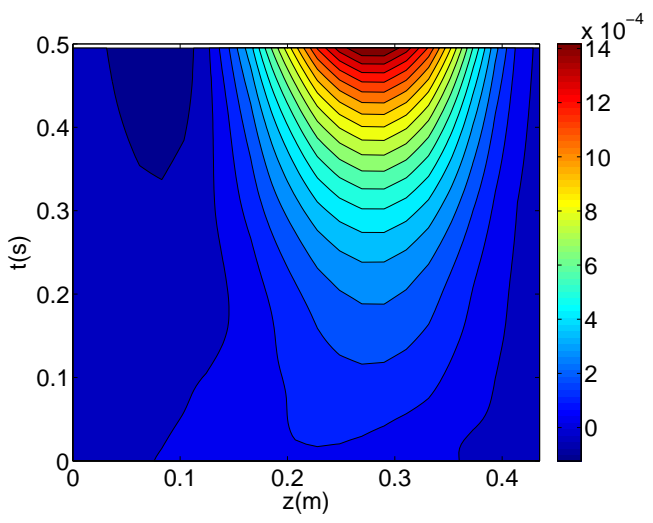

(b) $\mathrm{u}=7.5$

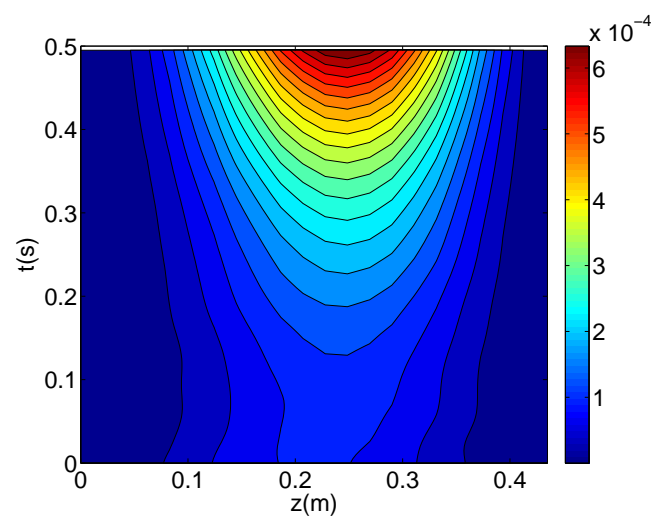

(d) $\mathrm{u}=9.5$

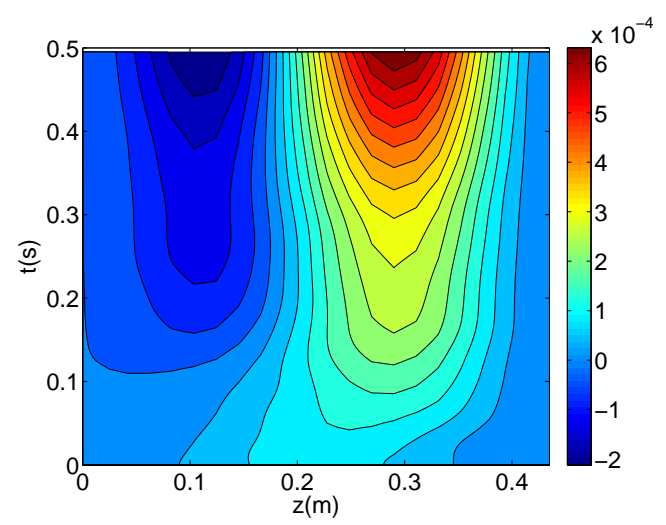

Figure 11: Center line displacement as function of time (up to $0.5 \mathrm{~s}$ ) displaying the initial repelling dynamics, for different flow velocities (from top left to down right: $u=7,7.5,8,9.5$ ).

\subsubsection{Attracting dynamics}

After the repelling region, the cylinder will be attracted to its stable buckled position. In the computations, the buckled state did not display a turning (out-of-plane) motion. It was however not quantified how much resistance the cylinder has against turning motions.

In the remainder of the text the attracting regime is considered to start at the moment the cylinder crosses its final steady state deformation and to stop at the end of the calculation. The displacement time history of each of the 20 points on the center line is fitted with $w=a \exp (-c t) \sin (\omega t+\phi)$. Differences in damping and frequency between the points is below $1 \%$ and thus very small. The mean damping and frequency are depicted in Figure 13.

The damping increases linearly in the stable region. In the buckled regime, however, the attracting transient is seen to have a damping higher and faster increasing than in the stable region. The different slope can be explained by investigating the flow profile around the buckled state. As expected from inviscid solutions the pressure is lower at the outer bend of the buckled cylinder in Figure 14. Concurrently, it displays an increase at the inner bend. The combination of these two leads to the centrifugal force often used in linear (and weakly nonlinear) descriptions. However, in contrast to the inviscid solution, the increase and decrease of the pressure are not symmetrical. The reason to this is that the flow separates (and two vortices exist) at the inner bend side of the cylinder, as is shown on the right side of Figure 14. As demonstrated earlier, the separation tends to increase the viscous forces significantly and change the contribution of viscous forces from a linear regime to a quadratic regime. It should also be noted that the separation point (and the change in dynamics it entails) is transported with the fluid flow.

The eigenfrequency of the first mode decreases in the stable region with increasing flow velocity. The onset of buckling is marked by a zero-frequency mode. Inside the buckling regime the frequency of the attracting transient 
(a) linear theory

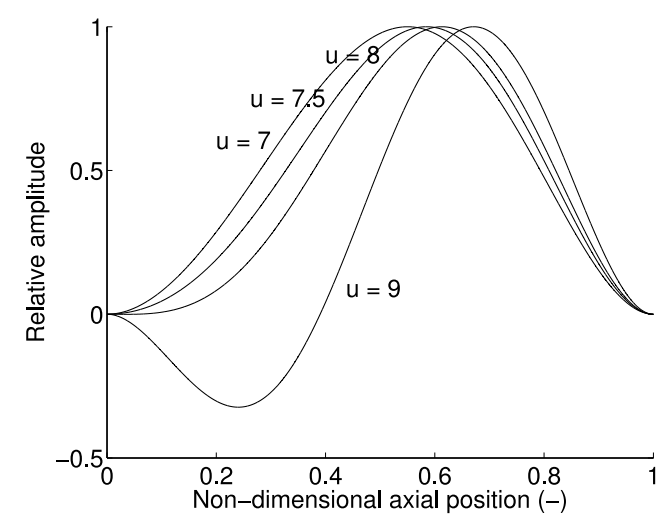

(b) simulated mode

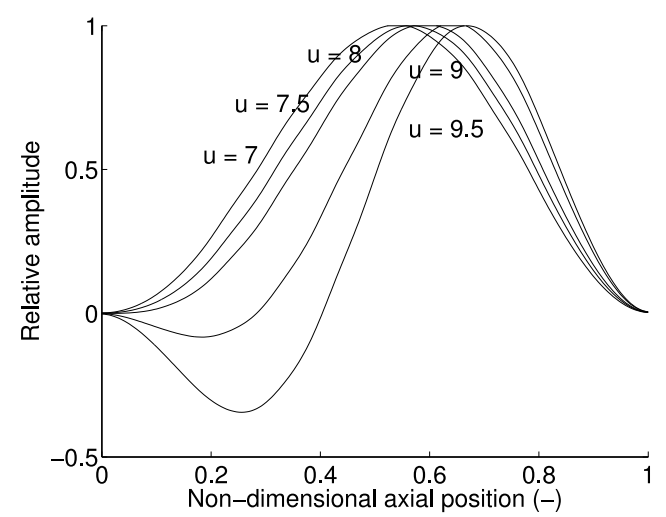

Figure 12: Mode shapes of the repelling transient according to linear theory (a) and according to the computations (b).
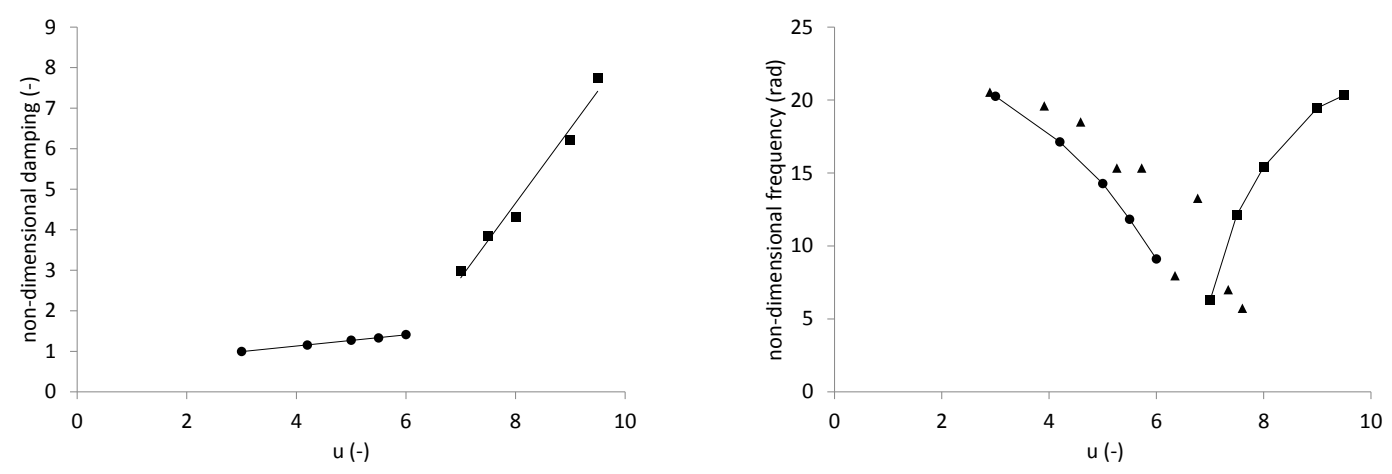

Figure 13: Damping and frequency of the ground mode in the stable ( $-\bullet$ ) and in the divergence ( $\rightarrow-)$ regime (around the buckled state). The experimental frequencies are denoted by triangles:

increases with increasing flow regime. This means that, if experiments are performed and the first mode frequency is measured, the onset of divergence will be marked by a V-shaped dip in frequency (see Figure 13).

Right before the cylinder restabilizes, marking the end of the divergence zone, it nearly regains its original frequency, which would occur if the flow was quiescent. It is however unlikely that this is a generally valid result.

\section{The post-divergence regime}

The weakly nonlinear theory of Modarres-Sadeghi et al. (2008) predicts that flutter around the buckled state will happen after the divergence region. Experimentally however, flutter became visible at much lower flow velocities and around the neutral center line. In previous, linear analysis, a condition for flutter was derived (Païdoussis (2004)), stating that the amount of work done on the system must be positive. For clamped-clamped boundary conditions, it leads to:

$$
-\left(C_{N}-C_{T}\right) \mathrm{v}^{2} \int_{0}^{L} \overline{\frac{\partial w}{\partial z} \frac{\partial w}{\partial t}} \mathrm{~d} z-\left(C_{N} \mathrm{v}+C_{D} \frac{4}{\pi}\right) \int_{0}^{L} \overline{\frac{\partial w^{2}}{\partial t}} \mathrm{~d} z>0,
$$

in which the overbar denotes long time averages. The presence of the different force coefficients in this equation shows that a good prediction of viscous forces is required in order to be able to assess whether flutter is going to happen or not. 
(a) Pressure contours

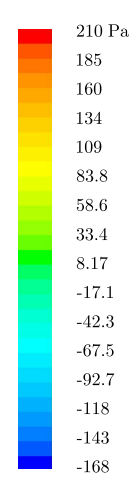

(b) In-plane velocity vectors
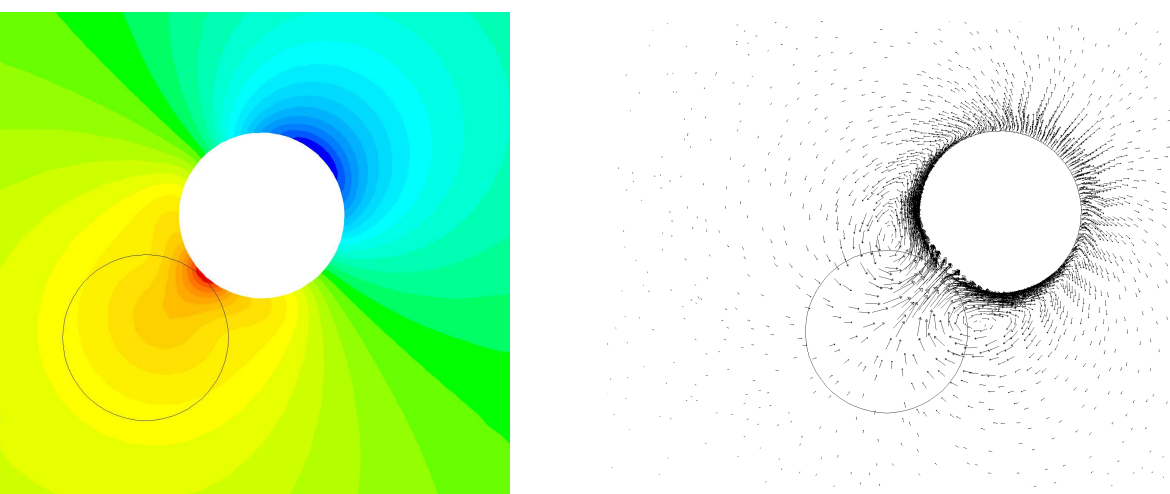

Figure 14: a) Pressure contours (Pa) and b) in-plane velocity vectors at a cross-sectional plane at a height of $0.23 \mathrm{~m}$ for a buckled cylinder with an average flow speed of $u=9.5$. The black circle denotes the original position of the cylinder. The overpressure coming from the centrifugal flow force is modified by the existence of the double vortex, visible on the right figure.
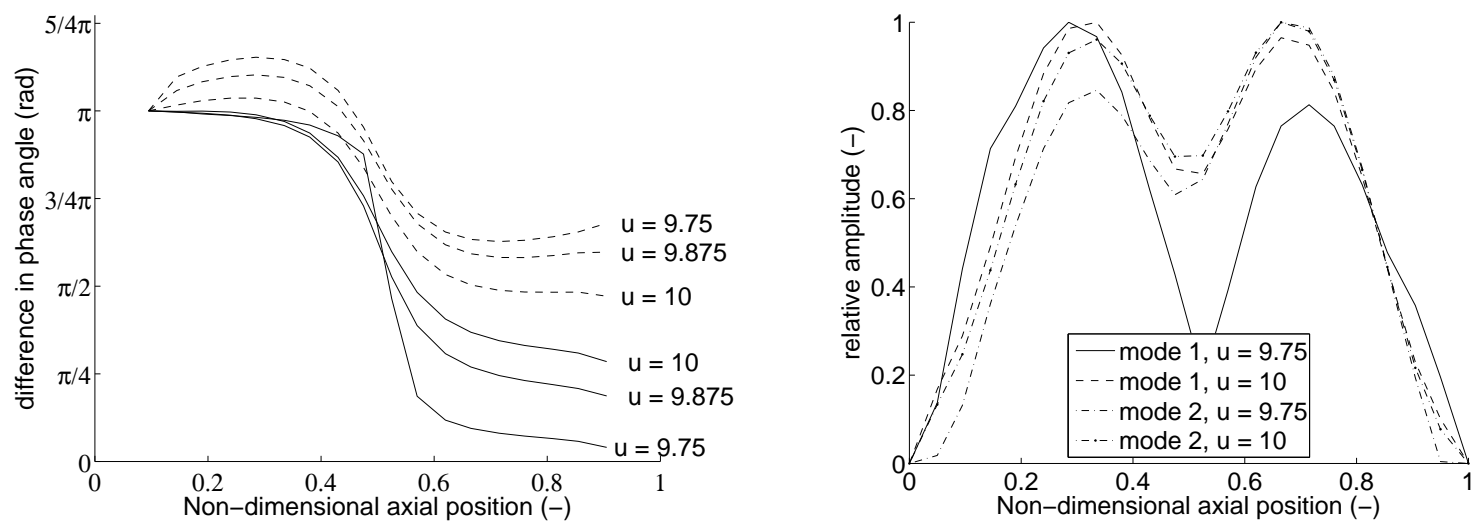

Figure 15: Evolution of the phase angle and amplitude of the two modes composing the transient in the restabilization regime with increasing flow velocity.

\subsection{Stabilization}

According to the present computational results, the structure restabilizes right after the divergence regime. In contrast to the results in the first stable regime, the transient is not composed of one single real mode, but of two complex modes (with position-dependent phase angle), which are shown in Figure 15. The phase of these modes has been shifted so that the most upstream points of both modes have an equal phase. The amplitudes have been normalized to attain a maximum value of one. Two modes are present because the imposed mode shape in the beginning of the simulation is not an eigenmode of the coupled system.

The mode denoted by a solid line in Figure 15, for convenience called mode one, shows at the lowest velocity a close agreement with an in-vacuum second mode; the right amplitude lobe is nearly $\pi$ rad out of phase with the left lobe. If the velocity increases, this phase difference decreases. In contrast to mode one, mode two is already a complex mode at low velocities. As the velocity increases, the phase of the two modes evolves to a similar profile.

The frequency and damping of these modes are plotted in Figure 16. While mode one is seen to increase its frequency and damping with increasing flow velocity, the second mode shows a decreasing frequency and damping trend. At slightly higher flow velocities, the cylinder starts to flutter, which means it has no (or negative) damping. These dynamics in between the divergence and the flutter regime take place in a very narrow velocity range and are therefore not easy to reproduce experimentally. In Figure 8 the experimental maximal amplitudes are however seen to decrease, which might thus be caused by the restabilization described above. 


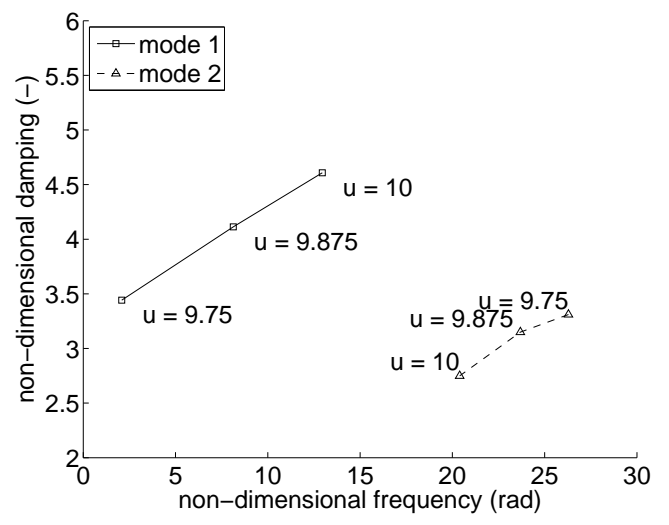

Figure 16: Argand diagram in restabilizing regime.

Table 5: Flutter frequency at different flow speed

\begin{tabular}{llllll}
\hline Non-dimensional flow velocity (-) & 10.125 & 10.25 & 10.5 & 11 & 11.25 \\
\hline Non-dimensional flutter frequency at near laminar inflow conditions (rad) & 17.8 & 17.4 & 12.7 & - & - \\
Non-dimensional flutter frequency at 5\% TI inflow conditions (rad) & 19.9 & 20.0 & 19.9 & 19.9 & 20.2 \\
\hline
\end{tabular}

\subsection{Flutter}

In Figure 16 the second mode, denoted by a dashed line, is seen to show a significant loss of damping with increasing non-dimensional velocity $u$. As expected, at slightly higher flow velocities, a flutter instability occurs. The post-processing of these results is slightly more difficult, as the fluttering motion did not necessarily remain in one plane, but instead could develop into a turning motion. A similar turning was also observed experimentally (Païdoussis et al. (2002)) for the flutter motion of a cylinder clamped upstream and free to move downstream.

To have results consistent with the experimental ones in Modarres-Sadeghi et al. (2008), the motion is projected on the yz-plane. The resulting transients halfway along the cylinder are plotted on Figure 17. The post-processing with projection on the Xz-plane yielded similar results.

In these graphs, it can be seen that only below a flow velocity of $u=11$ a flutter instability occurs. The resulting vibration does however not correspond to a purely sinusoidal motion. The main frequency of the fluttering motion is listed in Table 5.

The frequency is seen to decrease with increasing flow velocity. Concurrently, the maximal displacement, which is plotted in Figure 18 is seen to increase. The fluttering motion is, like the second stable mode in the previous paragraph, a complex mode. Its amplitude profile also consists of two lobes, with a more significant downstream lobe.

Next to results with near-laminar inlet conditions, the results of a separate series of calculations with higher inlet turbulence intensities are listed in Table 5. As Equation 9 shows that flutter of a clamped-clamped cylinder depends mainly on viscous forces, the inlet conditions, which were shown to affect these forces significantly in section 4 , are expected to have a large influence. The fluttering frequency is indeed slightly higher than in the case of near-laminar conditions. More importantly, the velocity range over which a fluttering motion is found is larger and the fluttering frequency is more stable against changes in flow velocity. It should also be noted that the relationship between flutter and turbulence might be affected by the turbulence model. As the angle and curvature dependent lift force is heavily influenced by the boundary layer, all parameters influencing the boundary layer are likely to influence the flutter regime.

The computations with a 5\% inlet turbulence intensity show a better agreement with the experimental values in Modarres-Sadeghi et al. (2008), as the predicted flutter frequency is $2.3 \mathrm{~Hz}$, whereas the experimental one is 1.8 $\mathrm{Hz}$. The maximal displacement halfway along the cylinder is underestimated with a computed value of $8 \mathrm{~mm}$, where the experiments measured a maximal value of $14 \mathrm{~mm}$. However, no experimental data about inlet turbulence was provided.

The present simulations predict flutter from a non-dimensional velocity of 10.125 , whereas experimentally it was 
(a) $\mathrm{u}=10.25$

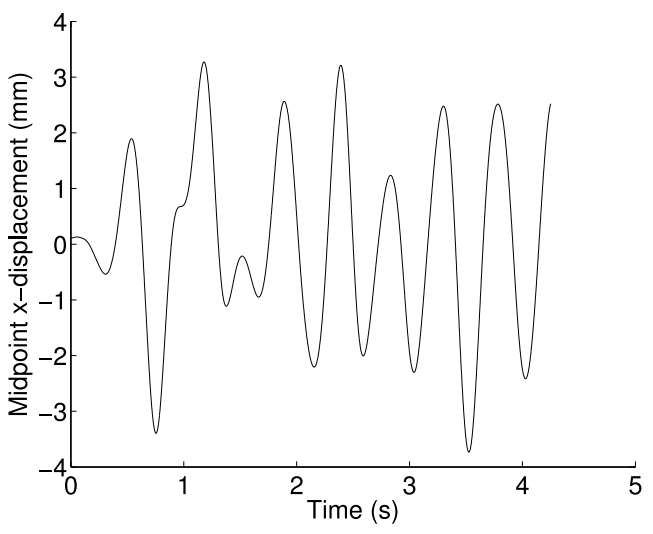

(c) $\mathrm{u}=11$

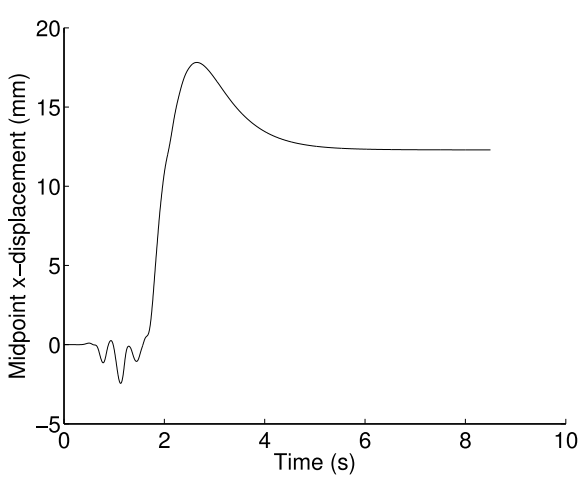

(b) $\mathrm{u}=10.5$

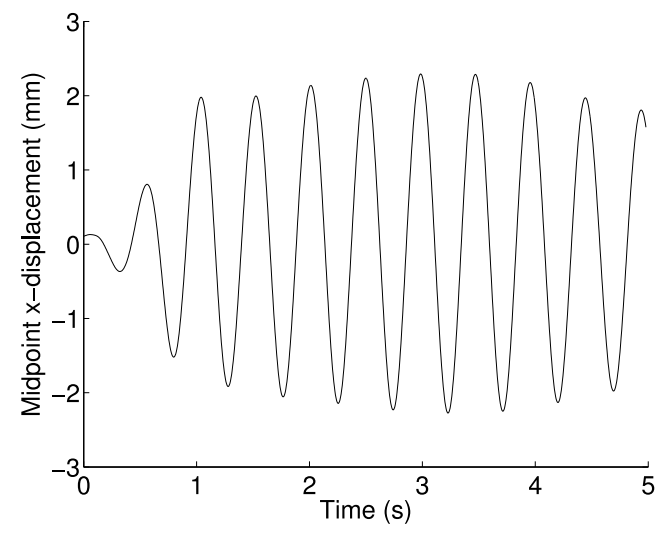

(d) $\mathrm{u}=11.25$

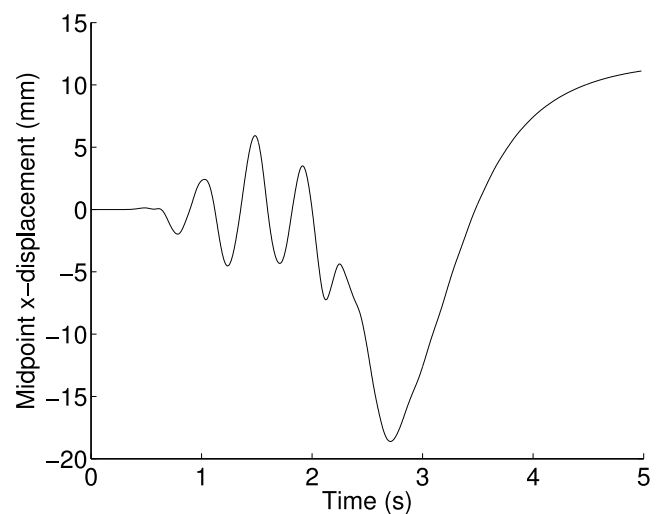

Figure 17: Transient halfway along the cylinder at a mean flow velocity of $\mathrm{u}=10.25,10.5,11,11.25$ with low levels of inlet turbulence. 


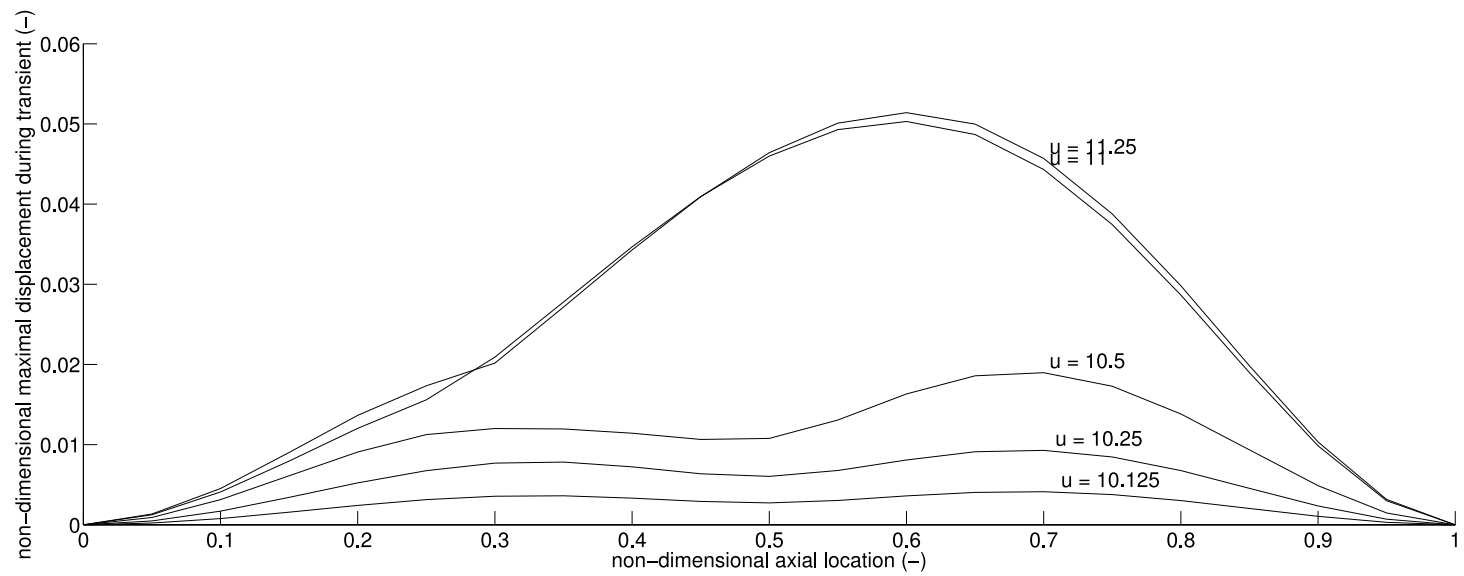

Figure 18: Maximal displacement of the fluttering motion (up to $\mathrm{u}=10.5$ ) and the transient towards the buckled state (from $\mathrm{u}=11$ ) as a function of the axial velocity.

observed at $\mathrm{u}=11.2$, which is a reasonably close prediction. The weakly non-linear theory (Modarres-Sadeghi et al. (2007)) predicted the fluttering motion only at $\mathrm{u}=15$. Moreover, it predicted flutter around a buckled position, while in both the simulations and the experiments it was observed that flutter occurred around the neutral axis.

Concluding this paragraph, the computations showed a reasonable agreement with experimental results. The inlet turbulence plays however a major role in the correct prediction of flutter. At low inlet turbulence, the fluttering motion was predicted to exist in a smaller flow velocity range.

\section{Conclusions}

In this paper, the dynamics of a flexible cylinder in axial flow have been studied using coupled CFD and CSM calculations. To verify the validity of the calculations, a specific case for which experimental data is available, is simulated.

The first part in this paper is concerned with the fluid forces predicted on rigid but deformed geometries. It was verified that the normal force on an inclined cylinder is linearly dependent on the angle of attack if it is small. Inside this region, the flow remained attached to the cylinder. At higher angles of attack, the flow detached from the cylinder modifying the pressure distribution and thus affecting the lift force behavior. Another consequence was the presence of two stationary vortices behind the cylinder. In the same part it was also shown that the lift force at small angles depends heavily on the inlet conditions. The lift force at high angles of attack was however less dependent on the inlet turbulence. Generally, the lift force increases with a more turbulent inlet. It should be noted that this conclusion is only valid for relatively unconfined flows, as confining effects will lead to fully developed flow conditions. The lift force coefficient was also shown to depend on the Reynolds number.

The same cylinder was also deformed by a circular arc to assess the influence of curvature on lift force. It was shown that at low curvature, the lift force is reasonably predicted by inviscid theory. If the local angle of attack is sufficiently large the flow detaches and a double vortex develops, which is transported downstream. As a result the lift force at higher curvature is lower than the one predicted by inviscid theory.

In the subsequent part, the dynamics of the system were computed by coupled CFD-CSM calculations. Consistent with earlier results, the damping increased and the frequency decreased with increasing flow velocity in the initial stable regime. The frequency decrease was furthermore in good agreement with experimental results. In the same velocity range, it was shown that small misalignment errors with the mean flow might be a significant contribution to the maximal displacement measured.

The predicted onset of divergence is found to be in good agreement with the results of a weakly nonlinear theory and experimental values, although the scatter on the latter is quite large. Increasing the angle of attack of the cylinder changed mainly the steady-state amplitude in the divergence region, which, for a straight cylinder, was within $20 \%$ of the experimental values. The repulsive dynamics were in qualitative agreement with dynamics predicted by linear 
theory. Around the stable buckled state, a first mode was found with increasing frequency. Its damping changed with a different slope than the corresponding mode in the stable regime, which could be attributed to a change in flow dynamics.

After the divergence region, a small zone of restabilization was found in which the second mode gradually shifted to a more complex mode with less damping, until it loses stability and developed a fluttering motion. This motion turned out to depend, consistent with the normal force computations earlier, on the inlet turbulence conditions. If the cylinder was slightly inclined it could skip this dynamic zone and develop only a divergence instability.

\section{Acknowledgments}

The authors gratefully acknowledge the funding by the Research Foundation-Flanders (FWO), through the Ph.D.fellowship of Jeroen De Ridder and the post-doc fellowship of Joris Degroote. The financial support of CWO for the research stay of Jeroen De Ridder at ENSTA is also gratefully acknowledged. The authors would like to thank Emmanuel de Langre and Pierre Moussou for fruitful discussions that motivated the study. The computational resources and services used in this work were provided by the VSC (Flemish Supercomputer Center), funded by Ghent University, the Hercules Foundation and the Flemish Government - department EWI.

Belanger, F., Delangre, E., Axisa, F., Paidoussis, M. P., Mateescu, D., 1994. Dynamics of coaxial cylinders in laminar annular-flow by simultaneous integration of the Navier-Stokes and structural equations. Journal of Fluids and Structures 8 (7), 747-770.

Chen, S. S., 1987. Flow-induced vibration of circular cylindrical structures. Hemisphere Pub. Corp.

de Langre, E., Paidoussis, M. P., Doare, O., Modarres-Sadeghi, Y., 2007. Flutter of long flexible cylinders in axial flow. Journal of Fluid Mechanics 571, 371-389.

De Ridder, J., Degroote, J., Van Tichelen, K., Schuurmans, P., Vierendeels, J., 2013. Modal characteristics of a flexible cylinder in turbulent axial flow from numerical simulations. Journal of Fluids and Structures 43, 110-123.

Degroote, J., Bathe, K. J., Vierendeels, J., 2009. Performance of a new partitioned procedure versus a monolithic procedure in fluid-structure interaction. Computers and Structures 87 (11-12), 793-801.

Divaret, L., Cadot, O., Moussou, P., Doaré, O., 2014. Normal forces exerted upon a long cylinder oscillating in an axial flow. Journal of Fluid Mechanics under consideration for publication.

Ersdal, S., Faltinsen, O. M., 2006. Normal forces on cylinders in near-axial flow. Journal of Fluids and Structures 22 (8), $1057-1077$.

Facci, A. L., Porfiri, M., 2013. Analysis of three-dimensional effects in oscillating cantilevers immersed in viscous fluids. Journal of Fluids and Structures $38(0), 205-222$.

Hoerner, S., 1965. Fluid-dynamic drag: practical information on aerodynamic drag and hydrodynamic resistance. Hoerner Fluid Dynamics

Lighthill, M. J., 1960. Note on the swimming of slender fish. Journal of Fluid Mechanics 9 (2), 305-317.

Lighthill, M. J., 1963. Oxford University Press, New-York, Ch. Introduction of boundary layer theory, pp. 46-113.

Lopes, J. L., Paidoussis, M. P., Semler, C., 2002. Linear and nonlinear dynamics of cantilevered cylinders in axial flow. part 2: The equations of motion. Journal of Fluids and Structures 16 (6), 715-737.

Menter, F. R., 1994. 2-equation eddy-viscosity turbulence models for engineering applications. AIAA Journal 32 (8), $1598-1605$.

Modarres-Sadeghi, Y., Païdoussis, M. P., Semler, C., 2007. The nonlinear behaviour of a slender flexible cylinder pinned or clamped at both ends and subjected to axial flow. Computers and Structures 85 (11-14), 1121-1133.

Modarres-Sadeghi, Y., Païdoussis, M. P., Semler, C., Grinevich, E., 2008. Experiments on vertical slender flexible cylinders clamped at both ends and subjected to axial flow. Philosophical Transactions of the Royal Society a-Mathematical Physical and Engineering Sciences 366 (1868), 1275-1296.

Païdoussis, M., 1973. Dynamics of cylindrical structures subjected to axial flow. Journal of Sound and Vibration 29 (3), 365-385.

Païdoussis, M. P., Grinevich, E., Adamovic, D., Semler, C., 2002. Linear and nonlinear dynamics of cantilevered cylinders in axial flow. part 1: Physical dynamics. Journal of Fluids and Structures 16 (6), 691-713.

Païdoussis, M., 2004. Fluid-Structure Interactions: Slender Structures and Axial Flow. Academic Press.

Perotin, L., Granger, S., 1997. A linearized unsteady model for computing dynamics of cylindrical structures subjected to nonuniform annular flows at high Reynolds numbers. Journal of Fluids and Structures 11 (2), 183-205.

Phan, C. N., Aureli, M., Porfiri, M., 2013. Finite amplitude vibrations of cantilevers of rectangular cross sections in viscous fluids. Journal of Fluids and Structures $40(0), 52-69$.

Rinaldi, S., Païdoussis, M. P., 2012. Theory and experiments on the dynamics of a free-clamped cylinder in confined axial air-flow. Journal of Fluids and Structures $28(0), 167-179$.

Sakuma, Y., Païdoussis, M. P., Price, S. J., 2008. Dynamics of trains and train-like articulated systems travelling in confined fluid-part 1: Modelling and basic dynamics. Journal of Fluids and Structures 24 (7), 932-953.

Semler, C., Lopes, J. L., Augu, N., Païdoussis, M. P., 2002. Linear and nonlinear dynamics of cantilevered cylinders in axial flow. part 3: Nonlinear dynamics. Journal of Fluids and Structures 16 (6), 739-759.

Sigrist, J.-F., Broc, D., Lainé, C., 2006. Dynamic analysis of a nuclear reactor with fluid-structure interaction: Part i: Seismic loading, fluid added mass and added stiffness effects. Nuclear Engineering and Design 236 (23), 2431-2443.

Singh, K., Michelin, S., de Langre, E., 2012. Energy harvesting from axial fluid-elastic instabilities of a cylinder. Journal of Fluids and Structures $30(0), 159-172$

Stokes, G. G., 1843. On some cases of fluid motion. Transactions of the Cambridge Philosophical Society 8, 30-37. 
Taylor, G., 1952. Analysis of the swimming of long and narrow animals. Proceedings of the Royal Society Series 214 (1117), $158-183$.

Triantafyllou, M. S., 1985. The dynamics of translating cables. Journal of Sound and Vibration 103 (2), 171-182. 